\title{
Electrochemical Synthesis of Coin Metal Nanoparticles in School Chemistry Education
}

\author{
Elena von Hoff, Stefanie Waitz, Felix Kollenda, Thomas Waitz* \\ Department of Chemistry Education, Georg-August-University Göttingen, Germany \\ *Corresponding author: twaitz@gwdg.de
}

\begin{abstract}
Within this article we present the synthesis of coin metal nanoparticles and nanoporous surfaces by cathodic corrosion as school experiments. We further present two experiments utilizing the obtained materials as catalysts; the catalytic decomposition of hydrogen peroxide and the platinum catalyzed chemiluminescence of lucigenin.
\end{abstract}

\section{Keywords: nanomaterials, catalysis, school chemistry}

Cite This Article: Elena von Hoff, Stefanie Waitz, Felix Kollenda, and Thomas Waitz, "Electrochemical Synthesis of Coin Metal Nanoparticles in School Chemistry Education." World Journal of Chemical Education, vol. 6, no. 1 (2018): 72-77. doi: 10.12691/wjce-6-1-11.

\section{Introduction}

For the synthesis of nanomaterials, a number of approaches exist which can be divided into two categories. In case of top-down processes the raw material (usually bulk material) is reduced in size using mechano-physical procedures, in order to receive particles on the nanometer scale. Whereas in bottom-up processes nanoparticles are formed by hydrolysis and condensation followed by nucleation (e.g. sol-gel processes).

The here described process of cathodic corrosion, intensively investigated by Yanson et al., can be assigned to the top-down category. Nanoparticles are obtained from a metal electrode through an electrosynthetic process and the electrode's surface is converted into a nanoporous material. Therefor a metal electrode is dipped into an electrolyte, while an inert electrode, e.g. graphite, serves as the counter electrode. By applying a low alternating voltage to the electrodes, nanoparticles form within seconds which agglomerate in the solution, creating an opaque dispersion. Suitable electrode metals are especially precious metals such as platinum, gold, copper and silver. For this particular synthesis, the electrolyte needs to be an aqueous solution containing cations. Typically, alkaline metal cations or non-metallic cations such as ammonium or tetraalkylammonium are used [1].

\section{The Cathodic Corrosion Mechanism}

The mechanism for the formation of nanoparticles using cathodic corrosion is not fully clarified in depth, however, the following processes are assumed to be involved which in particular are suitable as a teaching matter on secondary school level.

The applied alternating current polarizes the electrodes positively and negatively in turns. Especially the negative polarization is of importance, since the investigations by Yanson et al. have shown that nanoparticles are generated when the metal electrode continuously polarizes cathodically through an offset of the alternating voltage [1]. Because of the polarization, water is reduced to hydrogen and hydroxide at the phase boundary of the electrode (eq. 1):

$$
2 \mathrm{H}_{2} \mathrm{O}_{(l)}+2 e^{-} \rightarrow 2 \mathrm{OH}_{(a q)}^{-}+\mathrm{H}_{2(g)} .
$$

At the same time, the metal of the electrode is reduced because of the highly negative potential (eq. 2). The resulting anionic metal species (in the following equation represented by the symbol $\mathrm{Me}$ or $\mathrm{Me}_{\mathrm{x}+}^{\mathrm{y}+}$ respectively) are only stable in the anhydrous layer for a short time.

$$
x M e_{(s)}+y e^{-} \rightarrow M e_{x(a q)}^{y-} .
$$

Rodrigues et al. state that these anionic metal species are anionic metal clusters, which are mainly stabilized by the cations of the electrolyte [2]. If the clusters diffuse away from the electrode, they will be oxidized by water to their elementary state (eq. 3).

$$
\mathrm{Me}_{x(a q)}^{y-}+y \mathrm{H}_{2} \mathrm{O}_{(l)} \rightarrow \frac{y}{2} \mathrm{H}_{2(g)}+y \mathrm{OH}_{(a q)}^{-}+x \mathrm{Me}_{(s)} .
$$

This process is also assumed to take place, if the voltage applied to the electrode spontaneously decreases or even reverses during the phase shift of the alternating current. The anhydrous zone at the phase boundary between the electrode and the electrolyte won't be maintained and the anionic metal species will be oxidized. The resulting metal atoms and clusters aggregate within the electrolyte or at the surface of the electrode and form nanoparticles as well as the nanostructured surface of the electrode. During this process, the formation of larger nanoparticles is fostered by all factors increasing the stability and longevity of the anionic metal species. The factors include the type and concentration of the electrolyte's cations, the current and the voltage as 
well as the metal of the electrode, from which the nanoparticles are obtained [1]. A schematic drawing of the described processes on the particle level is shown in Figure 1.

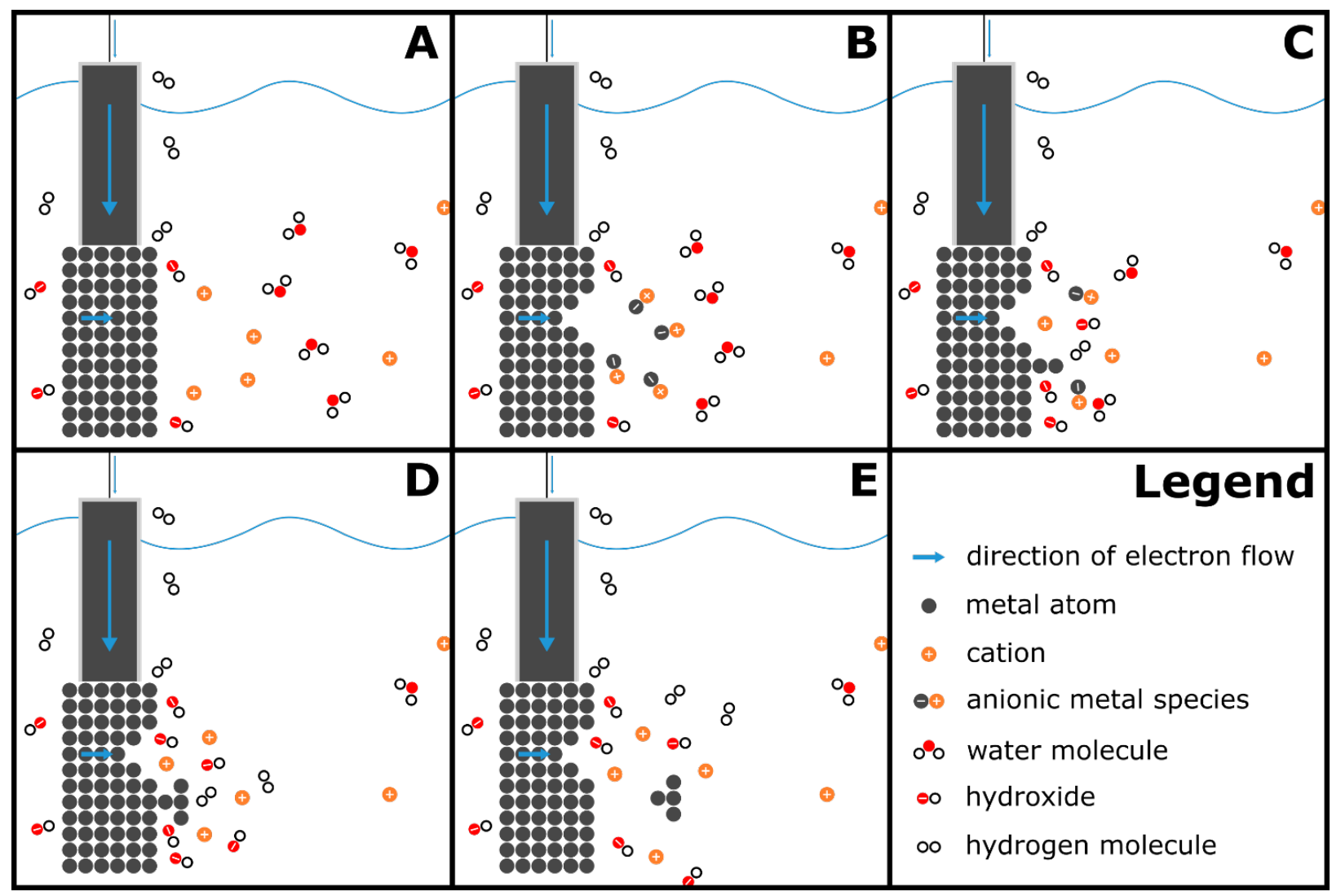

Figure 1. Model for the processes during the synthesis of the nanoparticles on the particle level. A: Due to the high cathodic potential at the metal electrode the water is reduced at the phase transition, resulting in an anhydrous layer around the electrode. B: The metal of the electrode along with the cations of the electrolyte form the anionic metal species. The latter are oxidized to elementary metal by water in C and D. E: The generated nanoparticles are released from the electrode

\section{Experiments}

\subsection{Synthesis of Nanoparticles by Cathodic Corrosion}

\section{Chemicals and equipment:}

Silver wire, copper wire, platinum wire, gold wire (Ø $1 \mathrm{~mm}), 250 \mathrm{~mL}$ beaker, approx. $200 \mathrm{~mL}$ warm $\left(\sim 60{ }^{\circ} \mathrm{C}\right)$ $1 \mathrm{M}$ sodium nitrate solution, stirring hot plate, stir-bar, alligator clip, graphite rod electrode, voltage source, 2 cables with $4 \mathrm{~mm}$ banana cord, 2 support stands, 2 support clamps, 2 clamp holders, glas rod, laser pointer. Experimental

An approx. $5 \mathrm{~cm}$ long piece of the metal wires is clamped into an alligator clip and, using a support stand and support clamp, is positioned thus that the wire is reaching vertically into a $250 \mathrm{~mL}$ beaker. The graphite rod electrode is clamped into the second support stand and positioned parallel to the wire in the same beaker. Using the cables, the electrodes are connected to the alternate current outputs of the voltage source. The voltage source is turned to $25 \mathrm{~V}$ and $12 \mathrm{~A}$ alternating current, making sure not to turn it on yet. Subsequently $200 \mathrm{~mL}$ warm $1 \mathrm{M}$ sodium nitrate solution are filled into the beaker and the electrodes are adjusted, so that the electrodes dip approx. $1 \mathrm{~cm}$ into the solution and are set about $1.5 \mathrm{~cm}$ apart. The voltage source is turned on for 2 minutes. After turning it off, the solution is stirred with a glass rod and the beam of a laser pointer is directed through the solution.

\section{Observation}

Immediately after turning on the voltage source a gas evolution can be observed at the electrodes. This is more pronounced at the metal electrodes and only slightly visible at the graphite electrode. Moreover, a smear formation originating from the metal electrodes can be observed. Figure 2 shows these observations for a setup including a gold electrode.

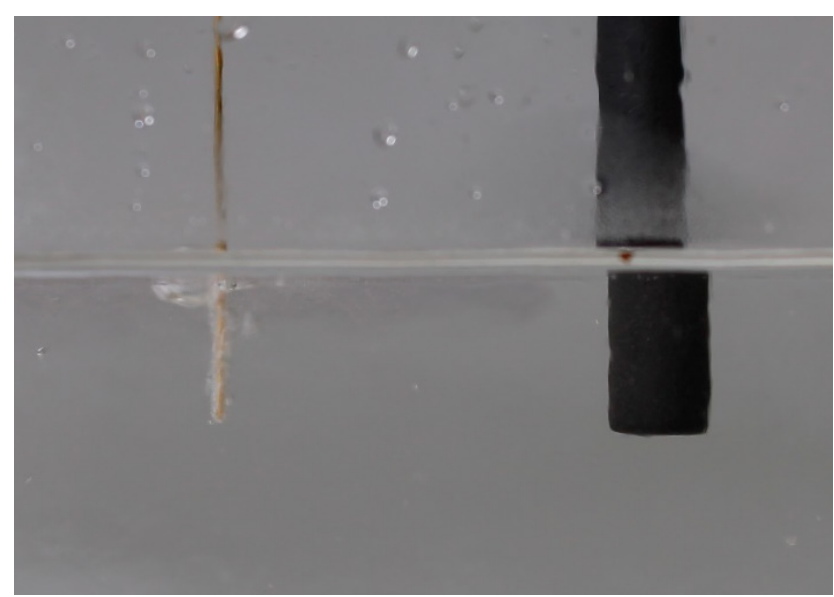

Figure 2. Gas evolution at a gold wire electrode immediately after turning on the voltage source. While a stream of gas is flowing upwards from the gold wire (left), only a weak gas evolution can be observed at the graphite electrode (right) 


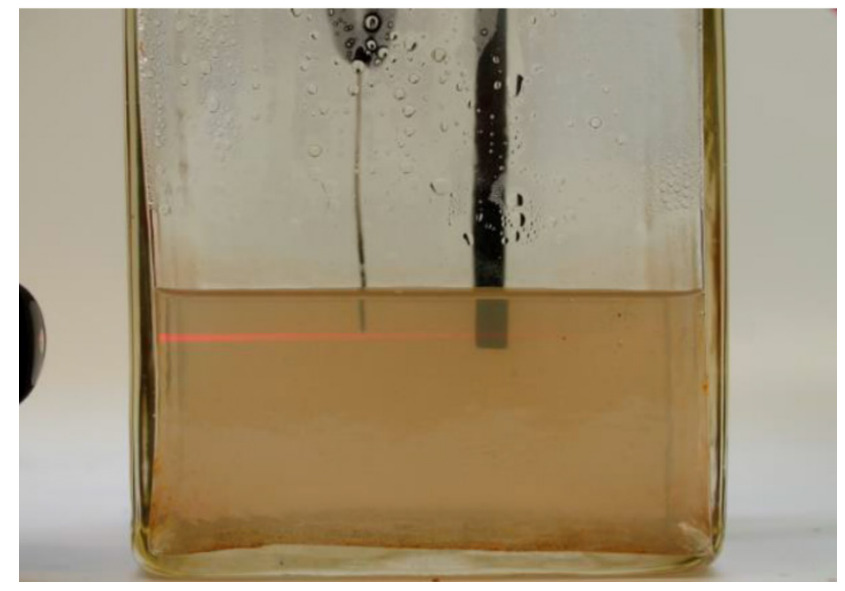

Figure 3. Reaction product of a cathodic corrosion using a copper electrode. A brown suspension can be observed, scattering the light of a laser beam

When directing a laser beam through the dispersion, the scattering of the laser beam is visible in all experimental setups. Figure 3 shows the visible laser beam in a dispersion, with a copper electrode used in the experiment.

The residues obtained after evaporation of the different dispersions were investigated by scanning electron microscope (SEM) analysis. In all cases a formation of (aggregated) nanoparticles was observed, varying in size depending on the metal used. When silver was used as the electrode material the particles sizes ranged from approx. $20-200 \mathrm{~nm}$, while the setup with the platinum electrode results in particles between 5 and $15 \mathrm{~nm}$ in size. Figure 4 shows exemplary SEM images of the obtained
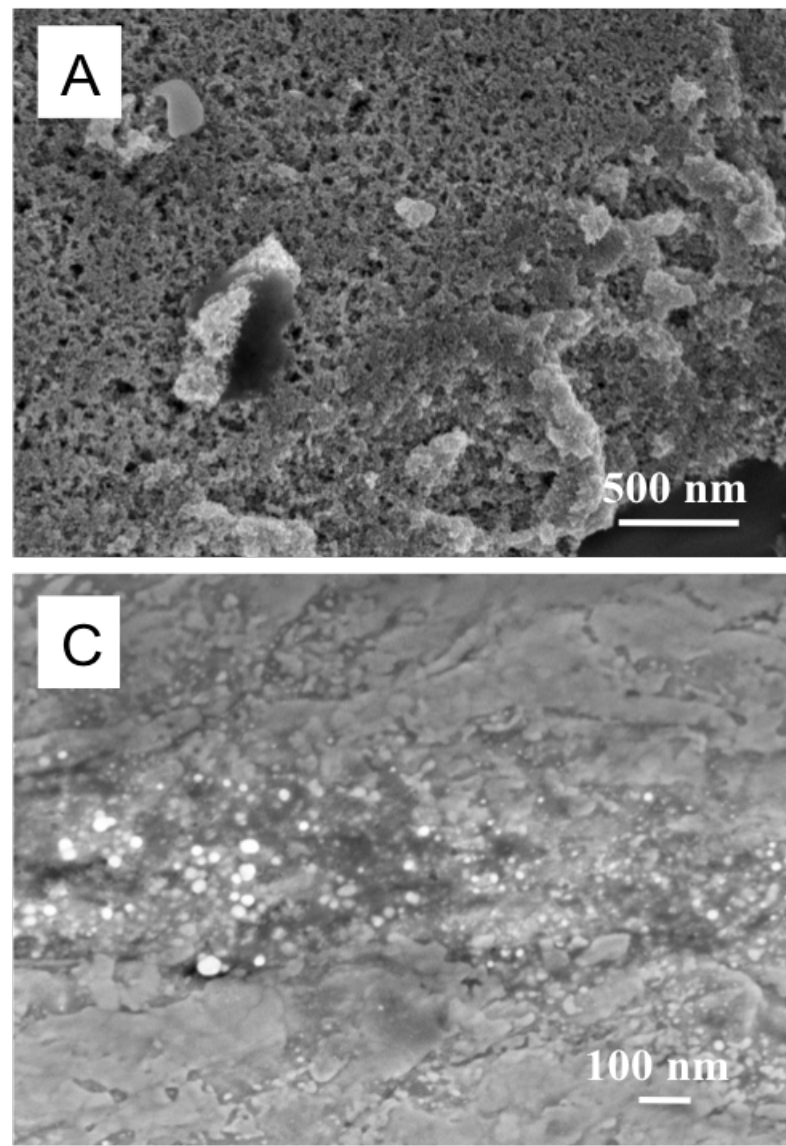

nanoparticles as well as of the wires before and after electrochemical treatment; the latter showing a nanoporous structure.

\section{Interpretation}

The gas evolution is caused by the reduction and oxidation of the water in the respective phases of the alternating current. In the anodic phase of the electrodes the water is oxidized (eq. 4):

$$
2 \mathrm{H}_{2} \mathrm{O}_{(l)} \rightarrow 4 \mathrm{H}_{(a q)}^{+}+\mathrm{O}_{2(g)}+4 e^{-} .
$$

In the cathodic phase, in turn, the water is reduced to hydrogen (eq. 5):

$$
2 \mathrm{H}_{2} \mathrm{O}_{(l)}+2 e^{-} \rightarrow 2 \mathrm{OH}_{(a q)}^{-}+H_{2(g)} .
$$

The resulting gases ascend along the metal wires as small bubbles. The faint gas evolution at the graphite electrode can be attributed to their porous structure and the resulting large surface area. The smear formation indicates the release of a newly formed substance into the medium. The inclusion of particles in the nano- to micrometer scale is confirmed by the scattering of the laser beam in the suspension (Tyndall effect). The SEM images of the evaporated dispersions also confirm the formation of nanoscale particles, which agglomerated into larger aggregates. Furthermore, the SEM analysis of the wires before and after the electrochemical treatment show the formation of nanoporous, disordered surface that can be ascribed to the corrosion of the respective materials; a mechanism with didactically reduced description can be found in section 1.
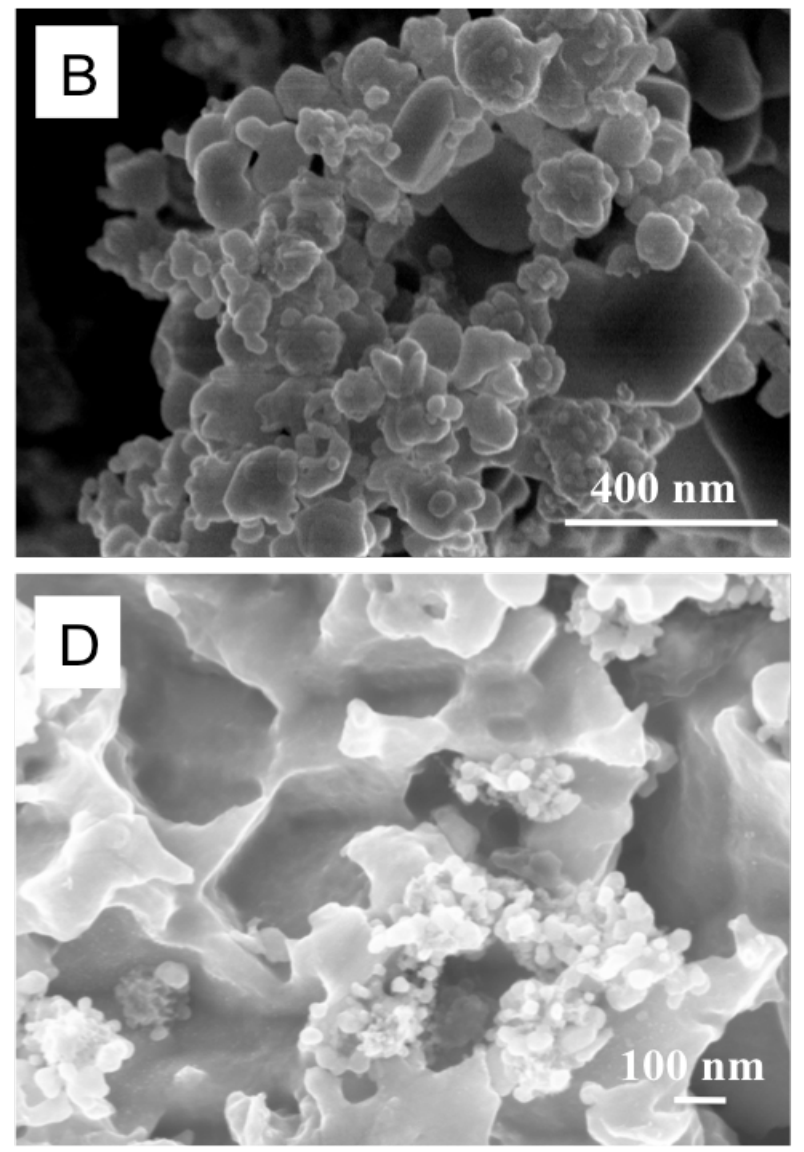

Figure 4. SEM images of the resulting nanoparticles (A and B) as well as the surface of a silver wire before (C) and after the electrochemical treatment (D) 


\subsection{Catalytic Decomposition of Hydrogen Peroxide}

\section{Chemicals and equipment:}

Copper-, silver-, gold- and platinum nanoparticle dispersions and respective untreated as well as electrochemically treated wires, concentrated hydrogen peroxide solution $(34.5 \%-36.5 \%)$, test tubes, splint, lighter, wire cutter, tweezers, petri dish.

Experimental:

A) Experiments with nanoparticle dispersions:

Approx. $1 \mathrm{~mL}$ of a copper-, silver-, gold- and platinum nanoparticle dispersion is filled into a test tube respectively and the same volume of concentrated hydrogen peroxide solution is added.

B) Experiments with metal wires:

A petri dish is filled with approx. $20 \mathrm{~mL}$ concentrated hydrogen peroxide solution. Subsequently approx. $1 \mathrm{~cm}$ long pieces of the untreated and electrochemically treated copper-, silver-, gold- and platinum wires are placed about $2 \mathrm{~cm}$ apart in the middle of the petri dish and the gas evolution is compared.

Observation:

Experiment A) After adding the hydrogen peroxide solution to the silver- and platinum nanoparticle dispersion an intense gas evolution is observed. Figure 5 shows this for a platinum nanoparticle dispersion. For copper- and gold nanoparticle dispersion, the reaction is less intense. When inserting a glowing splint into the test tubes, the splint glows intensely and catches fire.

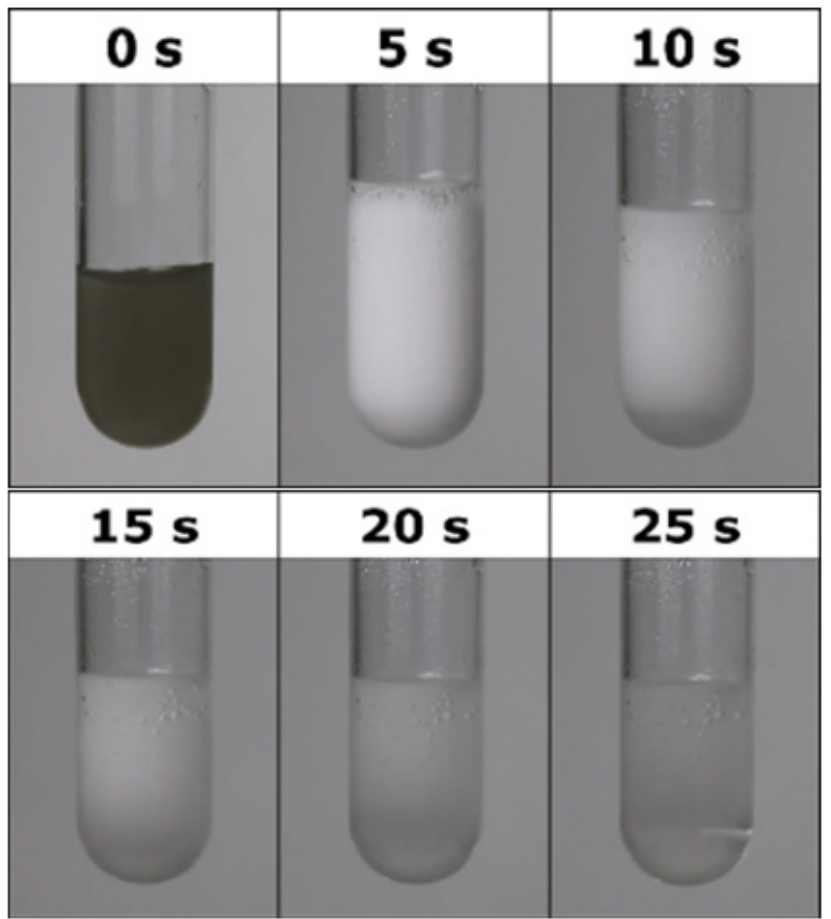

Figure 5. Reaction process of the gas evolution after adding concentrated hydrogen peroxide solution to a platinum nanoparticle dispersion.

Experiment B) At the untreated silver- and platinum wires a faint gas evolution is observed, while no gas evolution can be observed at the respective gold- and copper wires. At the electrochemically treated wires all metals show a distinct gas evolution. Comparing the gas evolution of the electrochemically treated wires with those of untreated wires, a significantly more pronounced gas evolution is observed at the silver- and platinum wires than at the untreated wires (Figure 6).

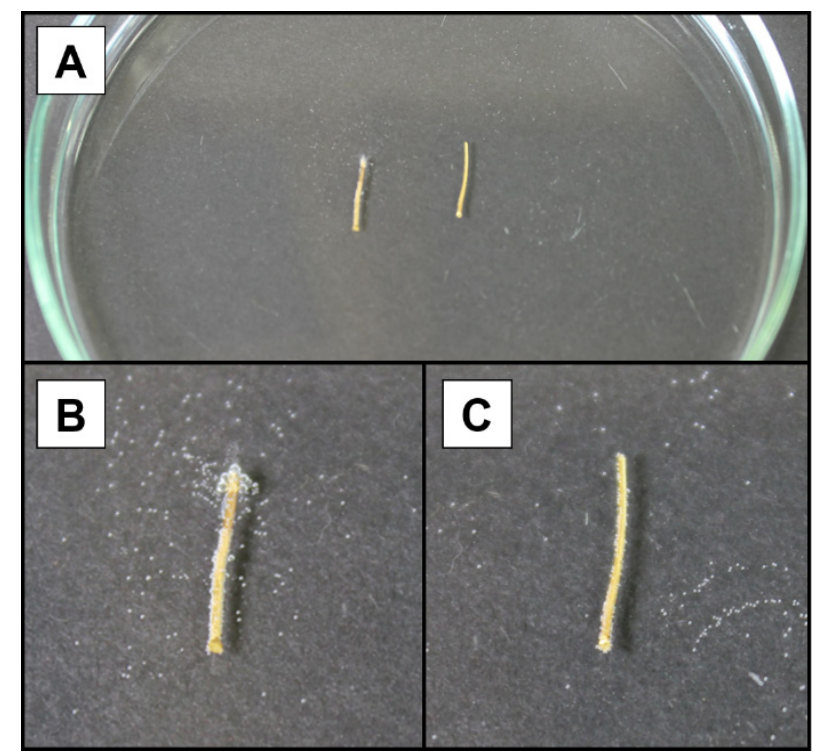

Figure 6. Comparison of the gas evolution between an electrochemically treated (A left, B) and an untreated gold wire (A right, C).

\section{Interpretation:}

Experiment A) The nanoparticles present in the aqueous phase catalyze the decomposition of hydrogen peroxide which can be derived from the positive oxygen detection. The catalytic effect of the nanoparticle dispersion however, varies in strength as shown by the varying intensity of the gas evolution.

Experiment B) The use of metal wires as catalysts shows that even the untreated metals are catalytically active, particularly silver and platinum. The electrochemically treated wires however are all visibly catalytically active, albeit with varying intensities. The increased activity can be explained with the enlarged surface area created by the cathodic corrosion.

The metal nanomaterials used in the experiments simultaneously serve as electron donors as well as acceptors. By accepting an electron from the metal's electron cloud the hydrogen peroxide molecule decomposes into hydroxide and a hydroxyl radical (eq. 6) $[3,4]$ :

$$
\mathrm{H}_{2} \mathrm{O}_{2(l)}+e^{-} \rightarrow \mathrm{OH}_{(a q)}^{-}+\mathrm{OH} \bullet{ }_{(a q)}
$$

The donation of an electron to the metal occurs through the deprotonated form of the hydrogen peroxide, forming a hydroperoxyl radical (eq. 7, 8):

$$
\begin{gathered}
\mathrm{H}_{2} \mathrm{O}_{2(l)} \rightleftharpoons \mathrm{HO}_{2(a q)}^{-}+\mathrm{H}^{+}{ }_{(a q)} \\
\mathrm{HO}_{2(a q)}^{-} \rightarrow \mathrm{HO}_{2}{ }_{(a q)}+e^{-}
\end{gathered}
$$

The resulting radicals in turn react with hydrogen peroxide. The hydroxyl radical reacts to form a hydroperoxyl radical as well as water, while the hydroperoxyl radical reacts forming a hydroxyl radical, water and oxygen gas (eq. 9, 10):

$$
\mathrm{OH} \cdot(a q)+\mathrm{H}_{2} \mathrm{O}_{2(l)} \rightarrow \mathrm{HO}_{2} \bullet{ }_{(a q)}+\mathrm{H}_{2} \mathrm{O}_{(l)}
$$




$$
\mathrm{HO}_{2} \bullet_{(a q)}+\mathrm{H}_{2} \mathrm{O}_{2(l)} \rightarrow \mathrm{OH}^{\bullet}(a q)+\mathrm{H}_{2} \mathrm{O}_{(l)}+\mathrm{O}_{2(g)} \cdot
$$

This chain reaction can be stopped by the reaction of the two radicals forming water and oxygen gas (eq. 11):

$$
\mathrm{OH} \cdot{ }_{(a q)}+\mathrm{HO}_{2} \cdot{ }_{(a q)} \rightarrow \mathrm{H}_{2} \mathrm{O}_{(l)}+\mathrm{O}_{2(g)} .
$$

Furthermore, the hydroxyl radical can react forming hydroxide by accepting an electron from the metal and the hydroperoxyl radical can react forming oxygen gas by donating an electron to the metal (eq. 12,13):

$$
\begin{gathered}
\mathrm{OH} \cdot{ }_{(a q)}+e^{-} \rightarrow \mathrm{OH}^{-}(a q) \\
\mathrm{HO}_{2} \cdot{ }_{(a q)} \rightarrow H^{+}{ }_{(a q)}+O_{2(g)}+e^{-} .
\end{gathered}
$$

\subsection{Platinum Catalyzed Chemilumi-nescence of Lucigenin}

Chemicals and equipment:

$5 \mathrm{mM}$ lucigenin solution, ethanol, $0.5 \mathrm{M}$ sodium hydroxide solution, platinum nanoparticle dispersion, $0.5 \mathrm{M}$ sodium nitrate solution, test tubes, Eppendorf pipettes and-vials, darkened room

Experimental:

A test tube is filled with $0.5 \mathrm{~mL}$ platinum nanoparticle suspension, $1.5 \mathrm{~mL}$ ethanol and $0.5 \mathrm{~mL}$ sodium hydroxide solution. Another test tube is filled with $3 \mathrm{~mL}$ lucigenin solution. In a darkened room the contents of the two tubes are merged.

\section{Observation:}

Approx. one minute after merging the contents of the two test tubes a blue-green glow can be observed that continues for a few minutes, as seen in Figure 7.

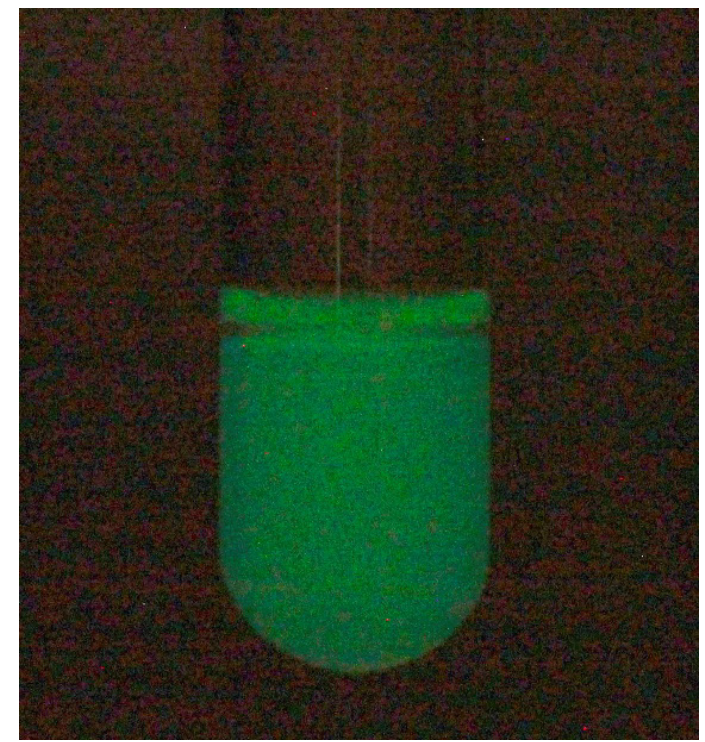

Figure 7. Platinum catalyzed chemiluminescence of lucigenin (photographic exposure time: 30 seconds).

\section{Interpretation:}

In alkaline solution and in the presence of a platinum/ethanol dispersion lucigenin dissociates into 10-methyl-9(10H)-acridone following several intermediate stages, with one of the two molecules in an excited state. When relaxing from the exited to the ground state a photon is emitted which causes the observed chemiluminescence (Figure 8) [5,6].

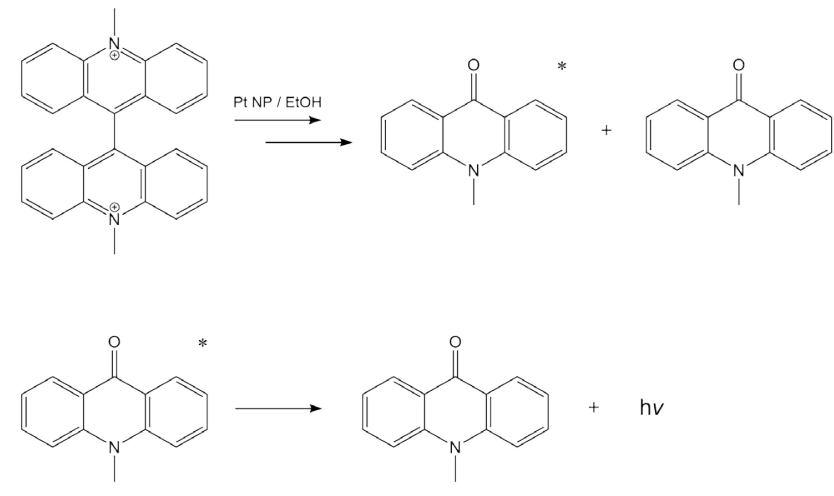

Figure 8.

\section{Educational Perspectives}

The presented experiments demonstrate an easy option to synthesize coin metal nanoparticles, either as porous wires or as particle dispersions. The underlying method of cathodic corrosion can be explained using an easy model on the particle level and are suitable for senior grades.

The described experiments offer the opportunity to investigate the catalytic properties of the resulting nanomaterials (porous wires and nanoscale particles). For the design of the experiments, using reference samples such as the untreated wires in the experiment for the catalytic decomposition of hydrogen peroxide should be considered. This allows easy comparisons between the catalytic activities of different materials $(\mathrm{Pt}, \mathrm{Cu}, \mathrm{Ag}, \mathrm{Au})$. During the critical consideration of the factors influencing the catalytic activity, not only the material but also the state of its surface should be discussed; the size of the electrochemically generated pores, for instance, influences the catalytic properties of the wires. Since the characterization of the pores is not necessarily possible in class, a cooperation with research institutes offers a great opportunity to bridge the gap between school and research. Questions arising during the course of the class, such as the texture of the surface of the wires, offer great opportunities to introduce scientific characterization methods such as SEM and to provide insights into the work as a scientist. In addition to the presented experiments, other experiments such as the catalytic oxidation of ethanol into acetaldehyde and its detection using fuchsine are possible.

\section{Acknowledgements}

We thank Mona Maaß for support in SEM analysis.

\section{References}

[1] Yanson, A. I., Rodriguez, P., Garcia-Araez, N., Mom, R. V., Tichelaar, F. D., Koper, M. T. M., „Cathodic Corrosion: A Quick, Clean, and Versatile Method for the Synthesis of Metallic Nanoparticles," Angew. Chem. Int. Ed., 50. 6346-6350. May 2011.

[2] Rodriguez, P., Tichelaar, F. D., Koper, M. T. M., Yanson, A. I., "Cathodic Corrosion as a Facile and Effective Method To Prepare Clean Metal Alloy Nanoparticles," J. Am. Chem. Soc., 133. 17626-17629. Oct. 2011. 
[3] Weiss, J, "The catalytic decomposition of hydrogen peroxide on different metals," Trans. Faraday Soc., 31. 1547-1557. Jul. 1935.

[4] McKee, D. W., "Catalytic Decomposition of Hydrogen Peroxide by Metals and Alloys of the Platinum Group," J. Catalysis, 14. 355-364. Aug. 1969.

[5] Maskiewicz, R., Sogah, D., Bruice, T. C., "Chemiluminescent Reactions of Lucigenin. 2. Reactions of Lucigenin with Hydroxide
Ion and Other Nucleophiles," J. Am. Chem. Soc., 101. 5355-5364. Aug. 1979.

[6] Duan, C. F., Cui, H., "Time-tunable autocatalytic lucigenin chemiluminescence initiated by platinum nanoparticles and ethanol," Chem. Commun., (18). 2574-2576. May 2009. 